\title{
The Method of Double Averaging: An Approach for Modeling Power-Factor-Correction Switching Converters
}

\author{
Siu-Chung Wong, Member, IEEE, Chi K. Tse, Fellow, IEEE, Mohamed Orabi, Member, IEEE, and \\ Tamotsu Ninomiya, Fellow, IEEE
}

\begin{abstract}
This paper describes the modeling of power-factorcorrection converters under average-current-mode control, which are widely used in switch-mode power supply applications. The objective is to identify stability boundaries in terms of major circuit parameters for facilitating design of such converters. The approach employs a double averaging procedure, which first applies the usual averaging over the switching period and subsequently applies generalized averaging over the mains period. The resulting model, after two averaging steps and application of a harmonic balance procedure, is nonlinear and capable of describing the low-frequency nonlinear dynamics of the system. The parameter ranges within which stable operation is guaranteed can be accurately and easily found using this model. Experimental measurements are provided for verification of the analytical results.
\end{abstract}

Index Terms-Averaged model, closed-form stability condition, double averaging, power factor correction (PFC), switching converters.

\section{INTRODUCTION}

$\mathbf{P}$ OWER-FACTOR-CORRECTION (PFC) boost stages are widely used in off-line switching power supplies. The basic configuration of a PFC boost stage consists of 1) an average-current-mode (ACM) control loop which "forces" the input current to follow the waveform of the input voltage; and 2) a slow output voltage feedback loop which controls the amplitude of the "shaped" input current according to the power demanded by the load. The aim of the control is to achieve a unity power factor at the input side and to maintain power balance while keeping the output voltage at a nearly constant level [1], [2]. Previously, several attempts have been made to model the dynamics of power converters based on dynamic phasors [3]-[5], which can be simplified in some specific applications to averaging and linearization [6]-[10]. However, because the actual process is

Manuscript received March 1, 2004; revised March 11, 2005 and July 6, 2005. This work was supported by the Hong Kong Research Grants Council under Grant PolyU 5237/04E, a Research Grant on "Control of Electromagnetic Environment in Low-Frequency Band Below $100 \mathrm{kHz}$ " of the future research promotion business in Japan provided by the Japan Society for Promotion of Science. This paper was recommended by Associate Editor I. A. Hiskens.

S.-C. Wong and C. K. Tse are with the Department of Electronic and Information Engineering, Hong Kong Polytechnic University, Hong Kong, China (e-mail: encktse@polyu.edu.hk).

M. Orabi is with the Electrical Engineering Department, Aswan Faculty of Engineering, South Valley University, Aswan, Egypt (e-mail: orabi@ieee.org).

T. Ninomiya is with the Department of Electrical and Electronic Systems Engineering, Kyushu University, Fukuoka 812-8581, Japan.

Digital Object Identifier 10.1109/TCSI.2005.855744 nonlinear, stability analyzes based on linearized models are very restricted and often provide stability information (e.g., parameter ranges for stable operation) which are not necessarily consistent with reality. In particular, low-frequency period-doubling phenomena in PFC boost preregulators have been reported by Orabi et al. [11]-[13]. Also, fast-scale instability has also been reported by Iu et al. [15]. In this paper we make use of a hierachical modeling approach, a basic form of which was proposed in [6], and apply generalized averaging together with a harmonic balance procedure to identify the low-frequency nonlinear dynamics of the PFC boost converter. This method results in a model that is capable of revealing the phenomena found in [11]-[13]. Essentially we apply averaging twice in succession, over the switching period and the mains period, to obtain a double-averaged model in the form of a system of simple continuous-time differential equations which can be readily analyzed and numerically simulated. Stability information generated from this model is accurate for the purpose of locating the ranges of parameters within which the PFC boost converter operates in its normally expected regime. This technique was also used in Gordillo et al. [14] for the study of the dynamics of a power factor preregulator under a special control. The work of Gordillo et al. [14], however, focuses on varying the output voltage. More comprehensive stability information that is presented in terms of major circuit parameters is unavailable. Furthermore, as is common to switching converters, operation may assume either continuous conduction mode (CCM) or discontinuous conduction mode (DCM). While much of the previous work has focused on the CCM case [11]-[15], very few has addressed the case of DCM [16]. Moreover, the problem of bifurcation of a supposedly CCM operation into DCM due to variation of some parameter(s) is a complex issue, which requires further investigation. In this paper we restrict ourselves to $\mathrm{CCM}$ operation, as it is the usual operating condition for most of medium to high power applications.

This paper is organized as follows. In Section II, we will explain the usual operation of the boost converter as employed in typical PFC applications, with emphasis on the salient operational conditions in the presence of a slowly varying input voltage. Our main proposal is described in Section III, where we explain the mathematical technique of doubling averaging, and apply it to produce a model for the PFC boost converter under average current-mode control. The derivation of the steady-state solution will be given in Section IV. Application of the doubleaveraged model for analysis of the stability of PFC converters 


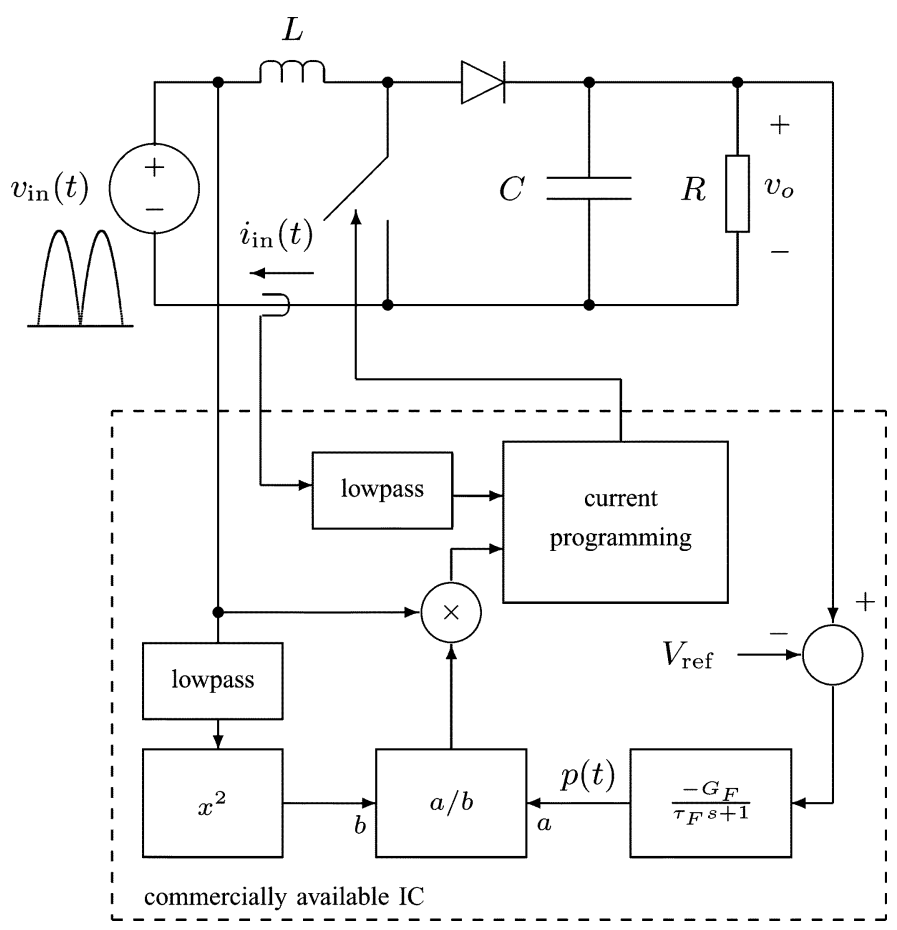

Fig. 1. Boost PFC converter under ACM control.

will be illustrated in Section V, where closed-form stability conditions are also derived. Finally, in Sections VI and VII, the stability information obtained from the model will be presented graphically and verified by experimental measurements taken from a practical industrial-standard PFC converter circuit.

\section{PFC BOOST CONVERTER UNDER ACM CONTROL}

The system under study is a boost converter under ACM control, as in typical PFC applications. As shown in Fig. 1, the input voltage is taken as a template for input current shaping. This templete is multiplied by a factor which effectively adjusts the amplitude of the input current. This amplitude control is derived from a nonlinear computational process involving a variable from the output feedback loop and the input voltage. To see how it works, we consider the basic requirement of the input current waveform, which is to be "ideally shaped" as

$$
i_{\text {in }}(t)=\frac{p(t) \sqrt{2}}{V_{\text {in }}}\left|\sin \omega_{m} t\right|
$$

where $\omega_{m}$ is the mains angular frequency, $V_{\text {in }}$ is the rms input voltage, and $p(t)$ is the output power which is given by a feedback circuit. Furthermore, if we use the input voltage as a template for shaping the input current, the amplitude must include an additional denominator $V_{\text {in }}$, as depicted in Fig. 1, i.e.,

$$
i_{\mathrm{in}}(t)=\frac{p(t)}{V_{\mathrm{in}}^{2}} v_{\mathrm{in}}(t)
$$

where $v_{\text {in }}(t)=\sqrt{2} V_{\text {in }}\left|\sin \omega_{m} t\right|$. As mentioned earlier, $p(t)$ is a feedback variable, which is derived from the output voltage. Obviously, $p(t)$ should vary very slowly relative to the mains half-cycle so that the sinusoidal waveform of the input current can be maintained. In practice, a low-pass type of feedback is

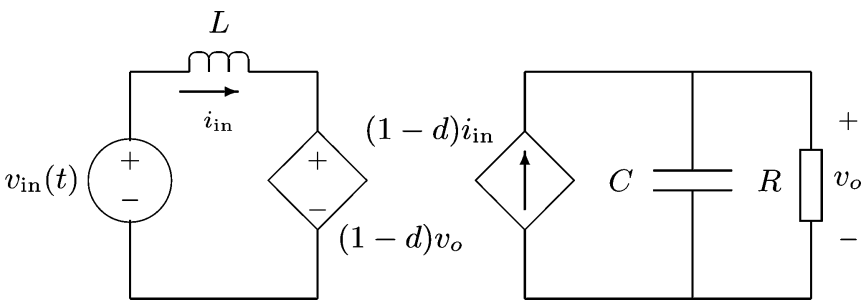

Fig. 2. First averaged model, where $d$ is implicitly defined by the ACM control.

applied to keep the output voltage nearly fixed. The usual control equation for $p(t)$ in the frequency domain is

$$
\frac{P(s)}{V_{o}(s)}=\frac{-G_{F}}{\tau_{F} s+1}
$$

where $P(s)$ and $V_{o}(s)$ are the frequency-domain variables of $\Delta p(t)$ and $\Delta v_{o}(t)$, respectively, and $G_{F}$ and $1 / \tau_{F}$ are the dc gain and cutoff frequency of the feedback circuit, respectively. Note that the equivalent time-domain equation is

$$
\tau_{F} \frac{d p(t)}{d t}+p(t)=-G_{F}\left(v_{o}-V_{\text {ref }}\right)
$$

where $V_{\text {ref }}$ is the reference output voltage. It is also worth mentioning that the overall dc gain in the loop must also include the factor $1 / V_{\text {in }}$ which is introduced by the divider at the input.

\section{Modeling By "Double Averaging"}

\section{A. First Averaging and Equation of Forced Oscillation}

For the purpose of studying the low-frequency dynamics, we may ignore the details within the switching cycle. This amounts to a standard averaging step that is to be taken over one switching period. The resulting model has appeared repeatedly in the literature of power electronics [17], [18]. We omit the details of the derivation here, but show the initial circuit model in Fig. 2. This model is incomplete because the duty cycle $d$ should be implicitly defined by the ACM control law. We first write down the Kirchhoff's law equations

$$
\begin{aligned}
& (1-d) v_{o}=v_{\text {in }}(t)-L \frac{d i_{\text {in }}}{d t} \\
& (1-d) i_{\text {in }}=C \frac{d v_{o}}{d t}+\frac{v_{o}}{R} .
\end{aligned}
$$

In the case of ACM control, the averaged inductor current has been programmed according to (1) or (2). From (5) and (6), and eliminating $d$, we get the following average power balance equation:

$$
\frac{C}{2} \frac{d v_{o}^{2}}{d t}=-\frac{v_{o}^{2}}{R}+i_{\text {in }} v_{\text {in }}-\frac{L}{2} \frac{d i_{\text {in }}^{2}}{d t} .
$$

For PFC applications, if the ACM achieves its objective of programming the current perfectly, the dynamics of the inductor can be ignored. However, failure of the ACM in programming the current is possible, and may lead to other forms of instability which are not studied here. Thus, we get

$$
\begin{aligned}
& \frac{C}{2} \frac{d v_{o}^{2}}{d t}=-\frac{v_{o}^{2}}{R}+2 p(t) \sin ^{2} \omega_{m} t \\
& \frac{C}{2} \frac{d v_{o}^{2}}{d t}=-\frac{v_{o}^{2}}{R}+p(t)\left(1-\cos 2 \omega_{m} t\right)
\end{aligned}
$$


for $n \pi \leq \omega_{m} t<(n+1) \pi, n=0,1,2, \ldots$. It should be noted that $p(t)$ is connected with $v_{o}$ via the feedback circuit. Therefore, (8) is a continuous-time differential equation in $v_{o}$.

Thus, the system under study can be described by the set of equations consisting of (4) and (8). To analyze this system, we will make use of the fact that it describes a system of forced oscillation, where the variables are "driven" periodically, containing frequency components up to twice the mains frequency.

\section{B. Second Averaging}

We now consider further application of averaging to the system of equations under study, which consists of (4) and (8). Specifically, we take moving average over the mains period [19]. For any variable $a(t)$, we may assume that it takes the following form:

$$
a(t)=A_{0}+A_{1} \sin \left(\omega_{m} t-\theta_{1}(t)\right)+A_{2} \sin \left(2 \omega_{m} t+\theta_{2}(t)\right) .
$$

We define each component of the moving average of $a(t)$, denoted by $[a(t)]_{k}$ or simply $[a]_{k}$ for $k=0,1,2$, by

$$
[a(t)]_{k}=[a]_{k}=\frac{\omega_{m}}{2 \pi} \int_{t-\frac{2 \pi}{\omega_{m}}}^{t} a(\tau) \exp \left(-j k \omega_{m} \tau\right) d \tau .
$$

For brevity and without confusion, we simply write $[a]_{k}$ as $a_{k}$. In other words, $a_{0}$ is the dc component of the moving average of $a(t), a_{1}$ is the component of the moving average of $a(t)$ at $\omega_{m}$, and $a_{2}$ is the component of the moving average of $a(t)$ at $2 \omega_{m}$. Thus, we may write

$$
\begin{aligned}
a(t)=a_{0}(t)+a_{1}(t) e^{j \omega_{m} t}+\left[a_{1}(t) e^{j \omega_{m} t}\right]^{*} \\
+a_{2}(t) e^{2 j \omega_{m} t}+\left[a_{2}(t) e^{2 j \omega_{m} t}\right]^{*}
\end{aligned}
$$

where superscript $*$ denotes complex conjugation. Upon expanding, we get

$$
\begin{aligned}
a_{0}(t) & =a_{0} \\
& =A_{0} \\
a_{1}(t) & =a_{1 r}+j a_{1 i} \\
& =-\frac{A_{1}}{2} \sin \theta_{1}-j \frac{A_{1}}{2} \cos \theta_{1} \\
a_{2}(t) & =a_{2 r}+j a_{2 i} \\
& =-\frac{A_{2}}{2} \sin \theta_{2}-j \frac{A_{2}}{2} \cos \theta_{2} .
\end{aligned}
$$

It is readily shown that the moving average of the time-derivative of each component is related to the time-derivative of the component by

$$
\begin{aligned}
{\left[\frac{d a(t)}{d t}\right]_{k} } & =\frac{\omega_{m}}{2 \pi} \int_{t-\frac{2 \pi}{\omega_{m}}}^{t} \frac{d a(\tau)}{d \tau} \exp \left(-j k \omega_{m} \tau\right) d \tau \\
& =\frac{d a_{k}(t)}{d t}+j k \omega_{m} a_{k}(t)
\end{aligned}
$$

where []$_{k}$ denotes consistently the $k$ th component of the moving average, with $k=0$ corresponding to the dc component, $k=1$ the $\omega_{m}$ component, and $k=2$ the $2 \omega_{m}$ component. Furthermore, the following relations are readily obtained:

$$
\begin{aligned}
{\left[a(t) \cos 2 \omega_{m} t\right]_{k}=} & \frac{1}{2}\left(a_{k-2}+a_{k+2}\right) \\
{\left[a^{2}(t)\right]_{0}=} & a_{0}^{2}+2\left|a_{1}\right|^{2}+2\left|a_{2}\right|^{2} \\
{\left[a^{2}(t)\right]_{1}=} & 2 a_{0} a_{1}+2\left(a_{1 i} a_{2 i}+a_{1 r} a_{2 r}\right. \\
& \left.+j\left(a_{1 r} a_{2 i}-a_{1 i} a_{2 r}\right)\right) \\
{\left[a^{2}(t)\right]_{2}=} & \left(a_{1}^{*}\right)^{2}+2 a_{0} a_{2} .
\end{aligned}
$$

Remarks: It should be noted that the essential procedure of taking the moving average differs from the basic double-averaging method of [6] where a constant steady-state point is assumed. Specifically, the method of [6] results in a model that reduces all variables varying at the mains frequency to fixed steady-state points. Thus, the subsequent small-signal analysis only reflects stability of the mains cycle but falls short of identifying oscillations at other subharmonics which have been found experimentally. As will be shown shortly, a moving-averaged model, coupled with the application of a harmonic balance procedure at any specific harmonic of interest, permits a quick identification of forced oscillation which may occur at half, quarter, etc. of the mains frequency. A similar application was also reported in Gordillo et al. [14], where bifurcation resulting from varying the output voltage was studied. However, practical stability information that is presented in terms of major circuit parameters is still unavailable.

\section{Derivation of the Double Averaged Model for PFC Boost Converter}

In this subsection we apply the foregoing averaging to (4) and (8). We expect to get three equations from each of (4) and (8), corresponding to the dc, $\omega_{m}$ and $2 \omega_{m}$ components. To avoid possible confusion due to mixup in subscripts, we define

$$
\left\{\begin{array}{l}
x=v_{o}(t) \\
y=p(t) .
\end{array}\right.
$$

In the following, the use of subscripts will be consistent with the description of the second averaging in Section III-B, i.e., subscript 0 for dc components, subscript 1 for mains frequency components, and subscript 2 for twice mains frequency components. Now, application of second averaging to (8) gives

$$
\begin{aligned}
& \frac{C}{2} \frac{d}{d t}\left(x_{0}^{2}+2 x_{1 r}^{2}+2 x_{1 i}^{2}+2 x_{2 r}^{2}+2 x_{2 r}^{2}\right) \\
& +\frac{\left(x_{0}^{2}+2 x_{1 r}^{2}+2 x_{1 i}^{2}+2 x_{2 r}^{2}+2 x_{2 r}^{2}\right)}{R} \\
& =y_{0}-y_{2 r} \\
& \frac{C}{2} \frac{d}{d t}\left(x_{0} x_{1}+x_{1 r} x_{2 r}+x_{1 i} x_{2 i}+j\left(x_{1 r} x_{2 i}-x_{1 i} x_{2 r}\right)\right) \\
& +\left(\frac{j \omega_{m} C}{2}+\frac{1}{R}\right)\left(x_{0} x_{1}+x_{1 r} x_{2 r}+x_{1 i} x_{2 i}\right. \\
& \left.+j\left(x_{1 r} x_{2 i}-x_{1 i} x_{2 r}\right)\right) \\
& =\frac{y_{1}}{2}-\frac{y_{-1}}{4} \\
& \frac{C}{2} \frac{d}{d t}\left(2 x_{0} x_{2}+\left(x_{1}^{*}\right)^{2}\right)+\left(j \omega_{m} C+\frac{1}{R}\right)\left(2 x_{0} x_{2}+\left(x_{1}^{*}\right)^{2}\right) \\
& =y_{2}-\frac{y_{0}}{2} \text {. }
\end{aligned}
$$




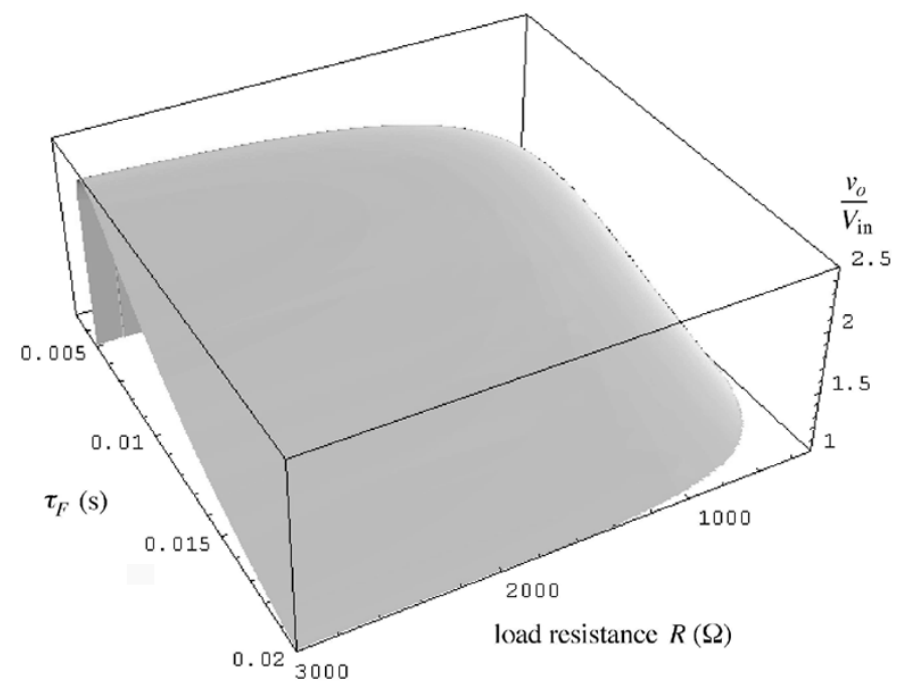

(a)

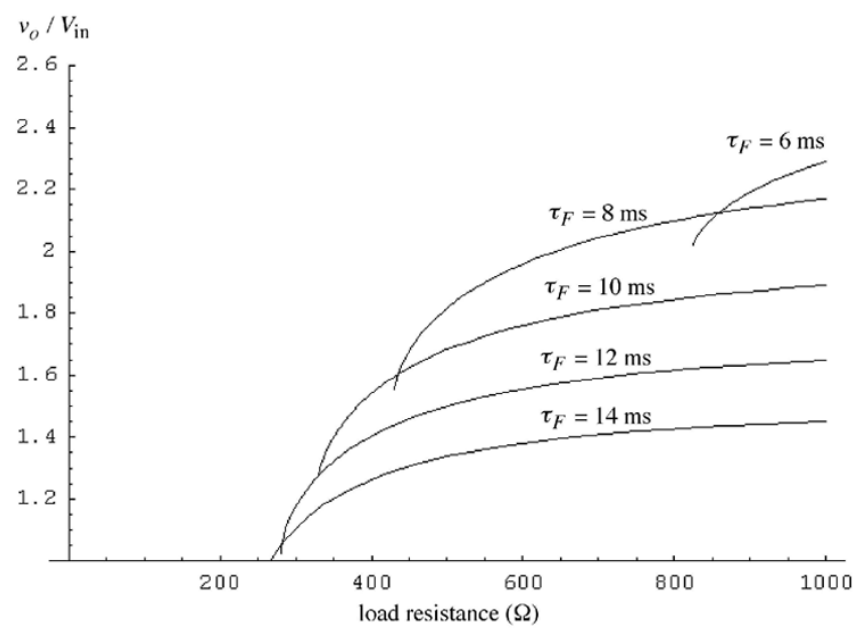

(c)

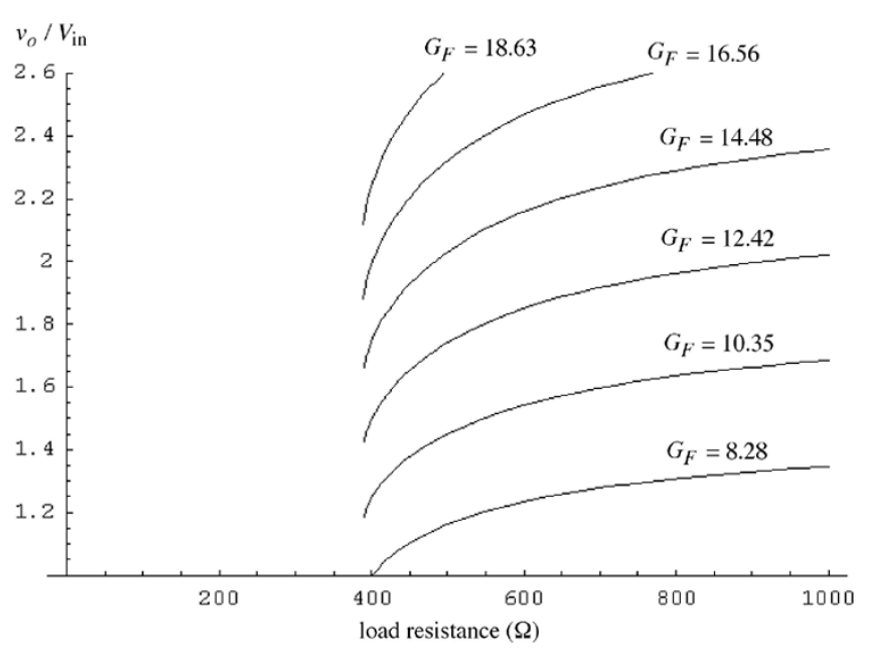

(b)

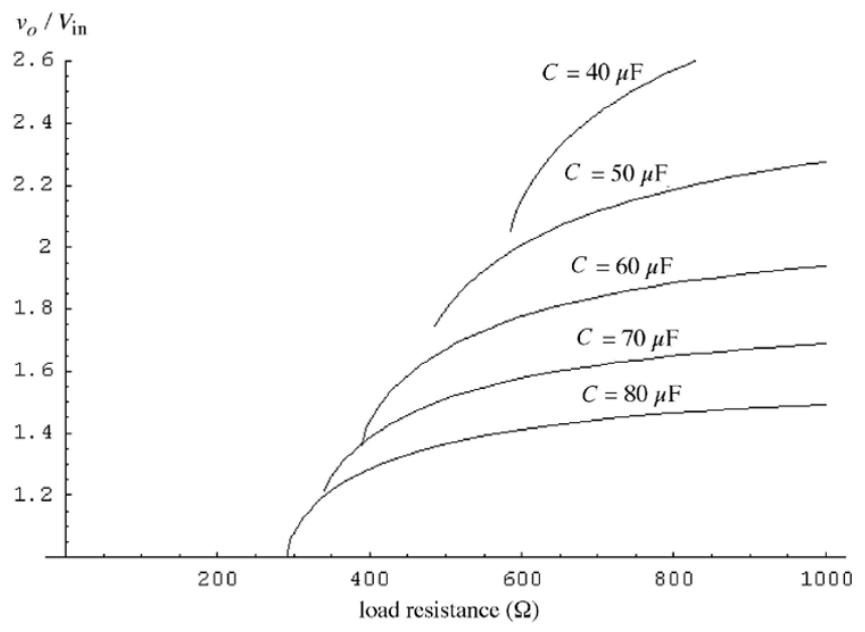

(d)

Fig. 3. Stability boundaries with: (a) $G_{F}=12.8 \mathrm{~A}, C=60 \mu \mathrm{F}$, and $\omega_{m}=2 \pi(60) \mathrm{rad} / \mathrm{s}$; (b) $\tau_{F}=8.6 \mathrm{~ms}, C=60 \mu \mathrm{F}$, and $\omega_{m}=2 \pi(60) \mathrm{rad} / \mathrm{s}$; (c) $G_{F}=12.8 \mathrm{~A}, C=60 \mu \mathrm{F}$, and $\omega_{m}=2 \pi(60) \mathrm{rad} / \mathrm{s}$; (d) $\tau_{F}=8.6 \mathrm{~ms}, G_{F}=12.8 \mathrm{~A}$, and $\omega_{m}=2 \pi(60) \mathrm{rad} / \mathrm{s}$. Region above the boundary surface or curve corresponds to stable operating region. At the boundary, period-doubling occurs to disrupt normal operation. Region under the boundary surface or curve corresponds to "unstable operation" in the sense that normal operation cannot be maintained.

Note that $y_{-1}$ is equal to $y_{1}^{*}$. Moreover, (4) is decomposed into

$$
\begin{aligned}
\tau_{F} \frac{d y_{0}}{d t}+y_{0} & =-G_{F}\left(x_{0}-V_{\text {ref }}\right) \\
\tau_{F} \frac{d y_{1}}{d t}+\left(j \omega_{m} \tau_{F}+1\right) y_{1} & =-G_{F} x_{1} \\
\tau_{F} \frac{d y_{2}}{d t}+\left(j 2 \omega_{m} \tau_{F}+1\right) y_{2} & =-G_{F} x_{2} .
\end{aligned}
$$

Thus, (21)-(26) are the double averaged equations for the PFC boost converter under study.

\section{Steady-State Solution}

To find the steady-state solution, we put all time-derivatives to zero, giving

$$
\begin{aligned}
& x_{1}=y_{1}=0 \\
& x_{2 r}=\left[\frac{V_{\text {ref }}-x_{0}}{2 \Delta}\right]\left[1+2 x_{0} \frac{1+4 \omega_{m}^{2} \tau_{F}^{2}}{G_{F} R}\right] \\
& x_{2 i}=\left[\frac{\omega_{m}\left(V_{\text {ref }}-x_{0}\right)}{\Delta}\right]\left[-\tau_{F}+x_{0} \frac{1+4 \omega_{m}^{2} \tau_{F}^{2}}{G_{F}}\right]
\end{aligned}
$$

$$
\begin{aligned}
& y_{2 r}=\left[\frac{V_{\text {ref }}-x_{0}}{\Delta}\right]\left[G_{F}+2 x_{0} \frac{1-2 \omega_{m}^{2} C R \tau_{F}}{R}\right] \\
& y_{2 i}=\left[\frac{V_{\text {ref }}-x_{0}}{\Delta}\right]\left[\omega_{m} C+\frac{2 \omega_{m} \tau_{F}}{R}\right] x_{0}
\end{aligned}
$$

where

$$
\begin{aligned}
\Delta=1+\frac{4\left(1-2 \omega_{m}^{2} C R \tau_{F}\right) x_{0}}{G_{F} R} \\
+\frac{4\left(1+\omega_{m}^{2} C^{2} R^{2}\right)\left(1+4 \omega_{m}^{2} \tau_{F}^{2}\right) x_{0}^{2}}{G_{F}^{2} R^{2}} .
\end{aligned}
$$

Putting the above equations in (21), and using (24), we get

$$
\begin{aligned}
& 8\left(1+\omega_{m}^{2} C^{2} R^{2}\right)\left(1+4 \omega_{m}^{2} \tau_{F}^{2}\right) x_{0}^{4} \\
& +8 G_{F} R\left[2-2 \omega_{m}^{2} C R \tau_{F}+4 \omega_{m}^{2} \tau_{F}^{2}\right. \\
& \left.+\omega_{m}^{2} C^{2} R^{2}\left(1+4 \omega_{m}^{2} \tau_{F}^{2}\right)\right] x_{0}^{3} \\
& +G_{F} R\left[-8 V_{\text {ref }}\left(1+\omega_{m}^{2} C^{2} R^{2}\right)\left(1+4 \omega_{m}^{2} \tau_{F}^{2}\right)\right. \\
& \left.+G_{F} R\left(9-12 \omega_{m}^{2} C R \tau_{F}+4 \omega_{m}^{2} \tau_{F}^{2}\right)\right] x_{0}^{2} \\
& +G_{F}^{2} R^{2}\left[G_{F} R+2 V_{\text {ref }}\left(-4+6 \omega_{m}^{2} C R \tau_{F}-4 \omega_{m}^{2} \tau_{F}^{2}\right)\right] x_{0} \\
& +G_{F}^{2} R^{2} V_{\text {ref }}\left[-G_{F} R+V_{\text {ref }}\left(1+4 \omega_{m}^{2} \tau_{F}^{2}\right)\right]=0
\end{aligned}
$$




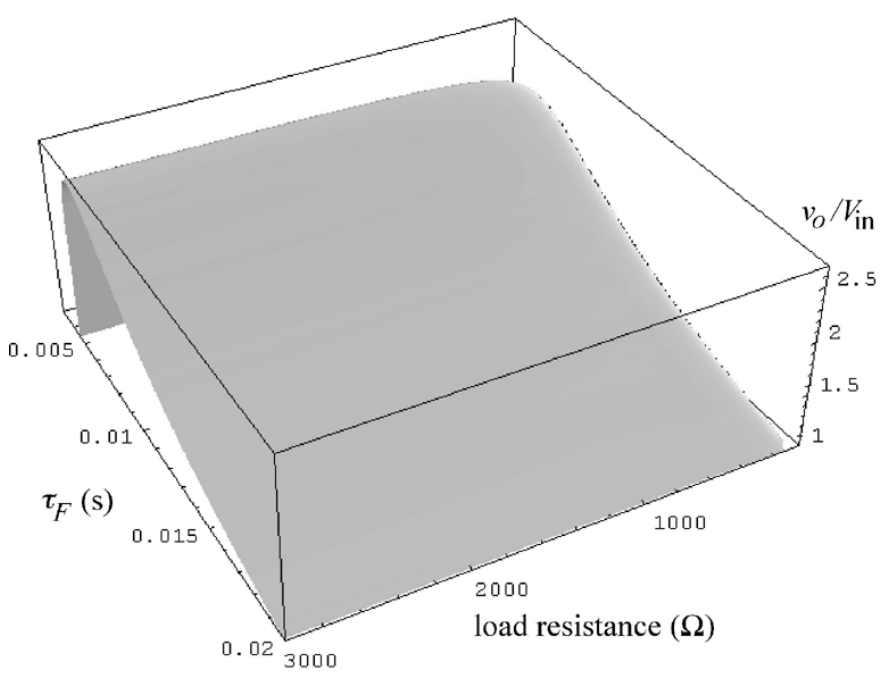

(a)

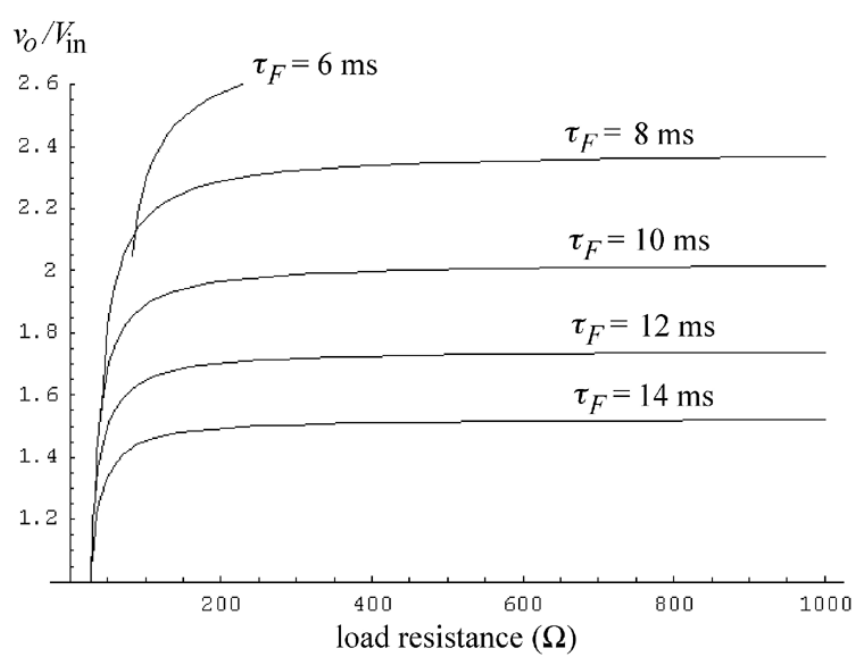

(c)

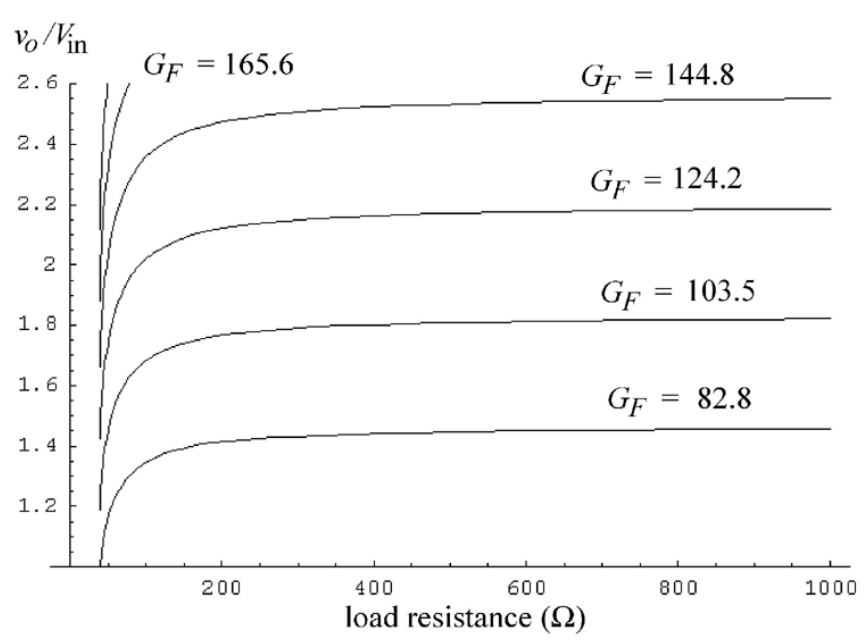

(b)

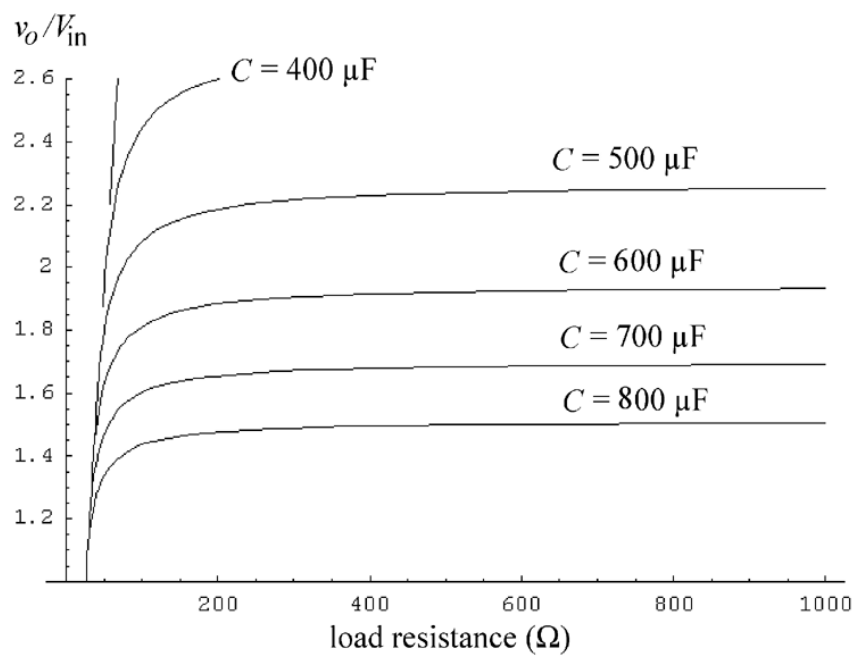

(d)

Fig. 4. Corresponding stability boundaries with: (a) $G_{F}=128 \mathrm{~A}, C=600 \mu \mathrm{F}$, and $\omega_{m}=2 \pi(60) \mathrm{rad} / \mathrm{s}$; (b) $\tau_{F}=8.6 \mathrm{~ms}, C=600 \mu \mathrm{F}$, and $\omega_{m}=$ $2 \pi(60) \mathrm{rad} / \mathrm{s} ;$ (c) $G_{F}=128 \mathrm{~A}, C=600 \mu \mathrm{F}$, and $\omega_{m}=2 \pi(60) \mathrm{rad} / \mathrm{s} ;$ (d) $\tau_{F}=8.6 \mathrm{~ms}, G_{F}=128 \mathrm{~A}$, and $\omega_{m}=2 \pi(60) \mathrm{rad} / \mathrm{s}$.

from which $x_{0}$ can be solved numerically. Note that $x_{0}$ is the steady-state dc component of the output voltage, which is close to $V_{\text {ref }}$.

\section{Application of Double Averaged Model to STABILITY ANALYSIS}

Our purpose in this section is to examine the stability of the system with respect to its operation at the forcing frequency $2 \omega_{m}$, which is twice the mains frequency. This is because the input is a rectified sine wave repeating at $2 \omega_{m}$, and all variables in the system are expected to repeat at this forcing frequency. Moreover, if the system is entrapped in an orbit of frequency $\omega_{m}$, i.e., at half the expected repetition frequency, ${ }^{1}$ the operation is considered undesirable for practical purposes since the device stresses would be drastically altered.

\footnotetext{
${ }^{1}$ In the literature of dynamical systems, such a change in operation is known as period doubling, and the transition from the normal operation to a double-period operation is typically a sudden transition. The usual term used to describe this transition is bifurcation [20], [21].
}

In fact, such "period-doubling" has been observed experimentally when certain parameters are changed irrespective of the stability of the $2 \omega_{m}$-orbit [11], [12]. It is therefore of practical importance to locate the occurrence of period-doubling in the parameter space. In our double-averaged model, this is equivalent to finding the condition under which the system is entrapped into an $\omega_{m}$-orbit.

Our approach to solve this stability problem is to consider the loop gain of $x_{1}$ (or $y_{1}$ ) in our double averaged model. Thus, from (22) and (25), and the steady-state values solved from (28) through (31), putting the time derivatives to zero, we get the loop gain for $x_{1}$ as shown in (34) at the bottom of the next page.

The system operates at the expected forcing frequency if it is not entrapped in the $\omega_{m}$-orbit, i.e., $T_{x 1}<1$. (It turns out that the condition $T_{x 1}>-1$ is irrelevant for practical sets of parameter values.) This condition is necessary for the system to operate in the expected regime. We may simplify (34) by putting $x_{0} \approx V_{\text {ref }}$, giving (35), shown at the bottom of the next page. We note that the condition of $T_{x 1}<1$ is equivalent to (36), also shown at the bottom of the next page. 


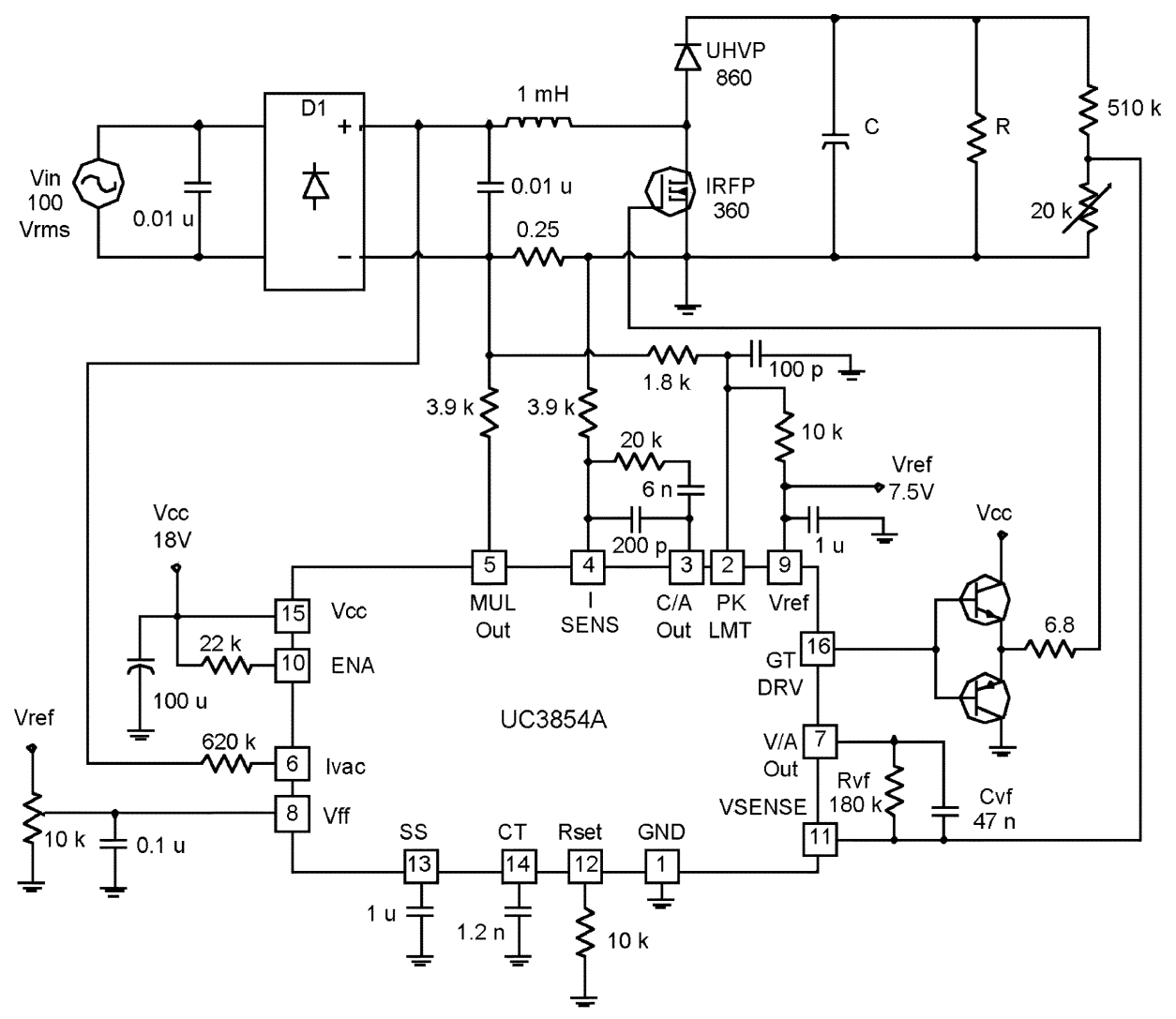

Fig. 5. Schematic of experimental PFC boost converter under average current-mode control.

This means that normal operation of the circuit at $2 \omega_{m}$ can be disrupted by period-doubling if there are parametric variations that violates (36). This is sometimes considered as instability from an engineering viewpoint as the system fails to operate in the expected manner. In particular, we observe the following.

1) There is a lower limit of $v_{o} / V_{\text {in }}$ below which the system cannot operate normally at the forced frequency $2 \omega_{m}$ (i.e, operating range is limited).

2) This lower limit of $v_{o} / V_{\text {in }}$ increases as the load resistance increases (i.e., normal operation is disrupted by period-doubling as output power decreases to a certain threshold).

3) This lower limit of $v_{o} / V_{\text {in }}$ generally increases as $G_{F}$ increases (i.e., operating range is reduced as the feedback gain increases).
4) This lower limit of $v_{o} / V_{\text {in }}$ generally increases as $\tau_{F}$ decreases (i.e., operating range is reduced as the feedback time-constant decreases).

5) This lower limit of $v_{o} / V_{\text {in }}$ generally increases as the output capacitance decreases (i.e., operating range is reduced as output capacitance decreases).

\section{GRAPHICAL REPRESENTATIONS OF STABILITY BOUNDARIES}

To help visualize the above results, we use (36) to plot a few indicative boundary surface and curves. Our purpose is to highlight the regions in the parameter space where normal operation is expected. Figs. 3 and 4 show several operation boundaries corresponding to some specific sets of parameters. On the boundary surface or curves, period-doubling occurs and normal operation

$$
\begin{aligned}
T_{x_{1}} & =\frac{G_{F} R}{2\left(4+\omega_{m}^{2} C^{2} R^{2}\right)\left(1+\omega_{m}^{2} \tau_{F}^{2}\right)\left(x_{0}^{2}-x_{2 r}^{2}-x_{2 i}^{2}\right)}\left\{\omega_{m} C R\left(\omega_{m} \tau_{F}\left(2 x_{0}-x_{2 r}\right)+x_{2 i}\right)-2\left(\omega_{m} \tau_{F} x_{2 i}+2 x_{0}+x_{2 r}\right)\right. \\
& \left.+\sqrt{-3\left(4+\omega_{m}^{2} C^{2} R^{2}\right)\left(1+\omega_{m}^{2} \tau_{F}^{2}\right)\left(x_{0}^{2}-x_{2 r}^{2}-x_{2 i}^{2}\right)+\left[-2\left(\omega_{m} \tau_{F} x_{2 i}+2 x_{0}+x_{2 r}\right)+\omega_{m} C R\left(\omega_{m} \tau_{F}\left(2 x_{0}-x_{2 r}\right)+x_{2 i}\right)\right]^{2}}\right\}
\end{aligned}
$$

$$
\begin{aligned}
T_{x_{1}} & =\frac{-\frac{3}{8} G_{F} R}{x_{0}\left[1-\frac{1}{2} \omega_{m}^{2} C R \tau_{F}+\frac{1}{2} \sqrt{1-4 \omega_{m}^{2} C R \tau_{F}-3 \omega_{m}^{2} \tau_{F}^{2}+\frac{1}{4} \omega_{m}^{2} C^{2} R^{2}\left(\omega_{m}^{2} \tau_{F}^{2}-3\right)}\right]} \\
x_{0}> & \frac{G_{F} R\left[\omega_{m}^{2} C R \tau_{F}-2+\sqrt{1-4 \omega_{m}^{2} C R \tau_{F}-3 \omega_{m}^{2} \tau_{F}^{2}+\frac{1}{4} \omega_{m}^{2} C^{2} R^{2}\left(\omega_{m}^{2} \tau_{F}^{2}-3\right)}\right.}{\left(4+\omega_{m}^{2} C^{2} R^{2}\right)\left(1+\omega_{m}^{2} \tau_{F}^{2}\right)}
\end{aligned}
$$




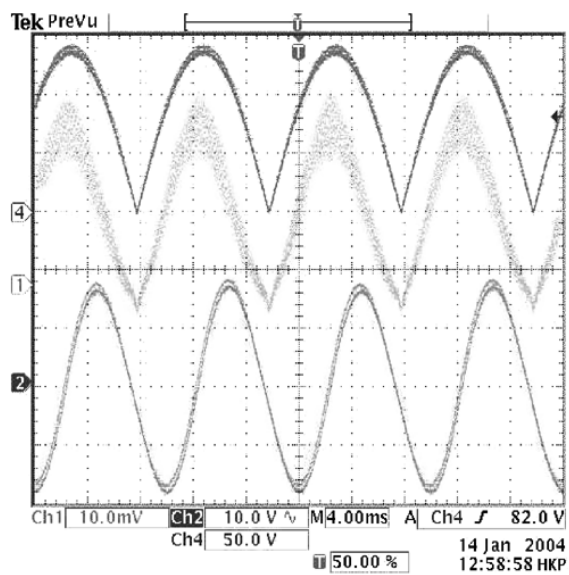

(a)

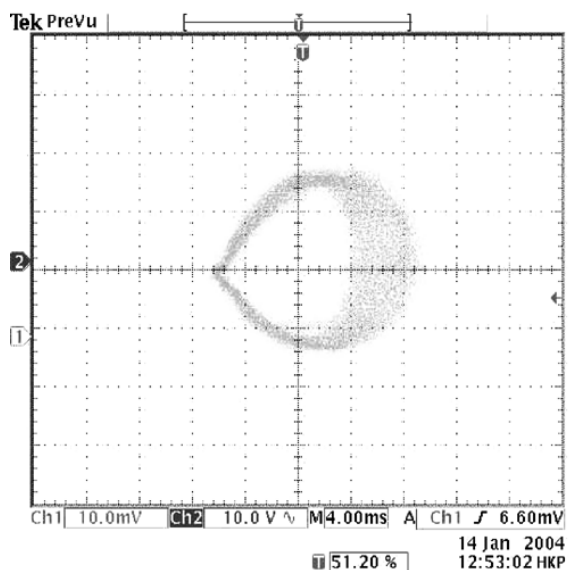

(d)

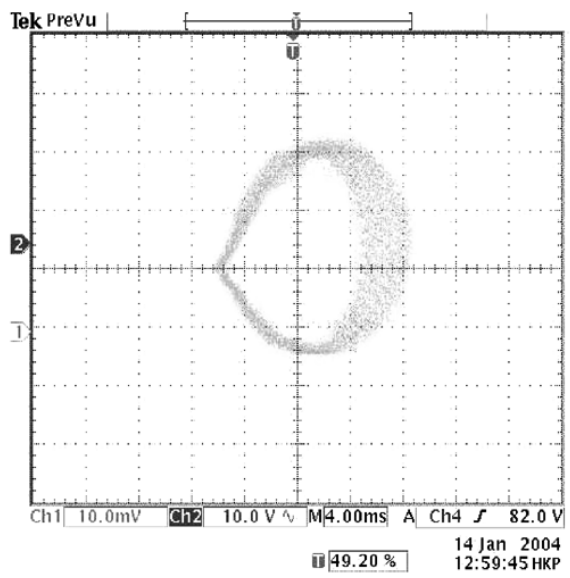

(b)

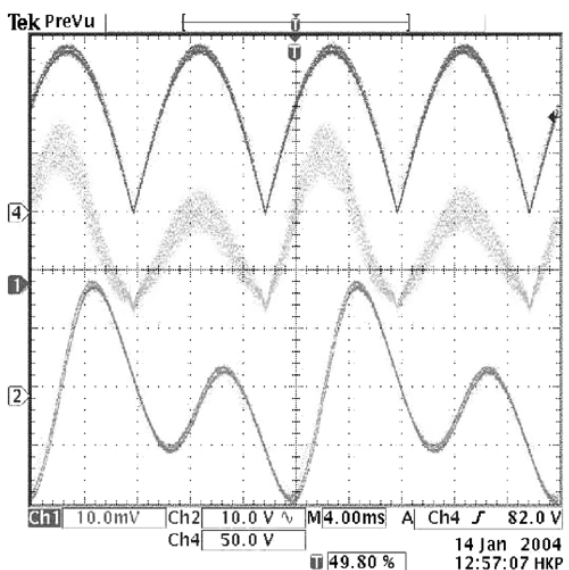

(e)

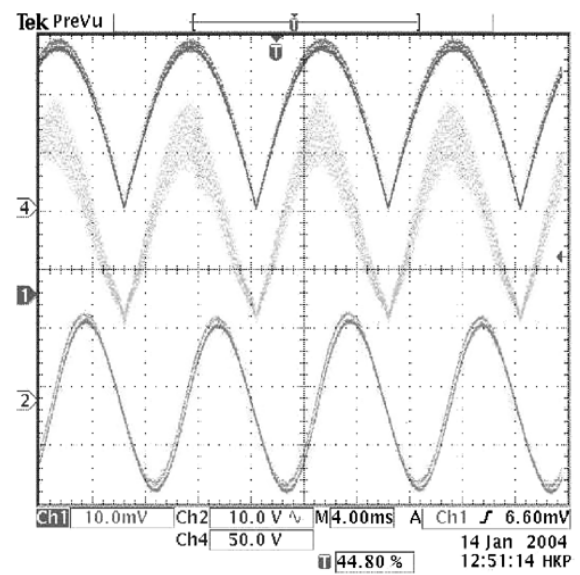

(c)

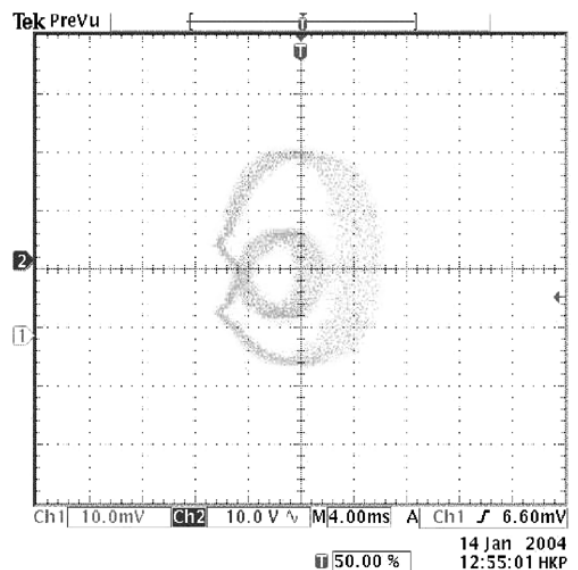

(f)

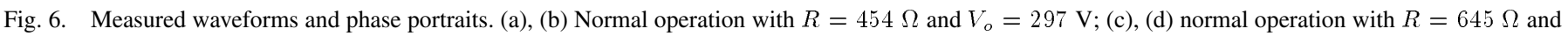

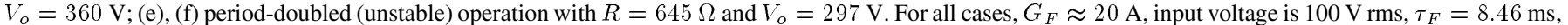

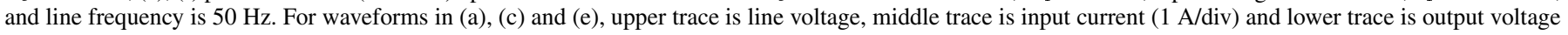
ripple. For phase portraits in (b), (d) and (f), $\mathrm{x}$ axis is input current and $\mathrm{y}$ axis is output voltage.

ceases. Region above the boundary corresponds to normal operation, where the system is driven periodically at twice the mains frequency. These results are consistent with our observation. Specifically, the operation is stable up to a certain threshold set of parameter values. In Fig. 3, the capacitor values are relatively small and the output ripple is therefore relatively large, causing instability when such a large ripple is being fed back. Moreover, in Fig. 4, the capacitor values are large (normal in practical sense), and instability can only occur for relatively high feedback gain values. Our analysis provides a quantitative tool for evaluating the stability boundaries. We will verify this tool in the next section by experiments.

\section{EXPERIMENTAL VERIFICATIONS}

An experimental PFC boost converter under average current mode control has been constructed for verification purposes. The complete schematic is shown in Fig. 5. The current-shaping control employs a standard 3854A integrated circuit controller. The line frequency is $50 \mathrm{~Hz}$. The input voltage is fixed at $100 \mathrm{~V} \mathrm{rms}$.

Experiments have been performed to verify the stability boundaries predicted in Sections V and VI. First, for a quick illustration, typical waveforms and phase portraits for normal operation and period-doubled (unstable) operation are shown in Fig. 6.

Our main objective is to locate the parameter points where period-doubling occurs. For convenience we replot the stability curves along with the experimental data in Fig. 7. We clearly see that the experimental data are in good agreement with the analytical results.

A few practical points are worth noting here.

1) One of the determining parameters is $v_{o} / V_{\text {in }}$, as shown in our analytical study. However, for measurement convenience, we fix the input voltage in our experiments and vary the output voltage.

2) In our analytical model, the feedback loop is defined by two parameters, namely $G_{F}$ and $\tau_{F}$. In order to find the corresponding value of $G_{F}$ for the experimental circuit, we have to carefully identify all gains that may possibly contribute to $G_{F}$ since the model assumes no gain in the multiplier-divider block. In our experiment, we find this gain by direct measurement.

3) Our definition of $\tau_{F}$ is the real time constant. Thus, its reciprocal should be compared directly with the angular mains frequency in $\mathrm{rad} / \mathrm{s}$ instead of the frequency in 


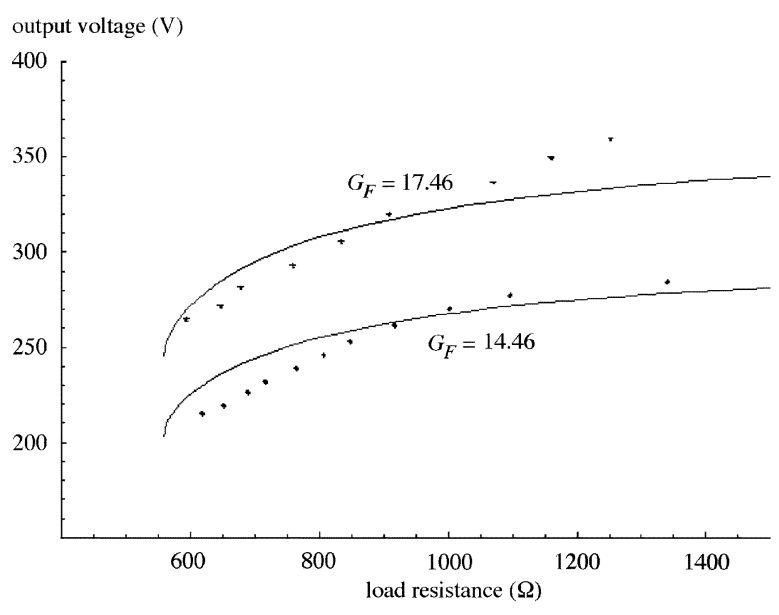

(a)

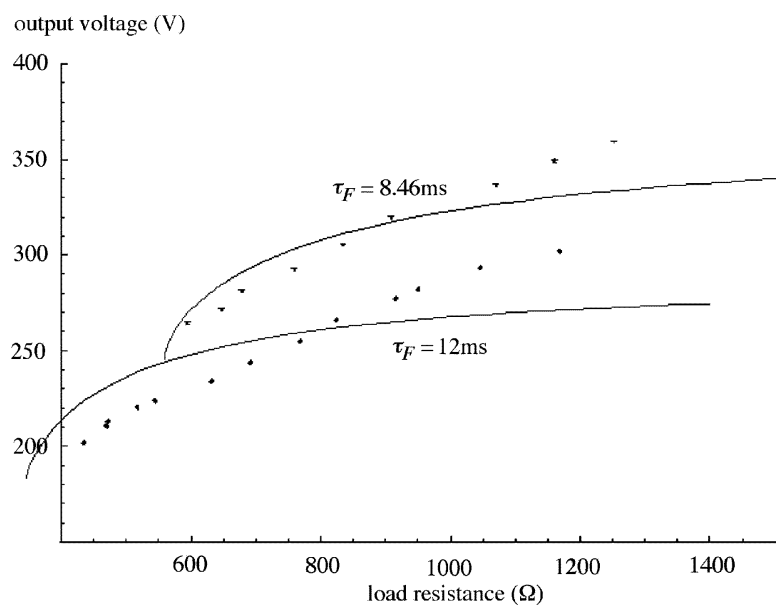

(b)

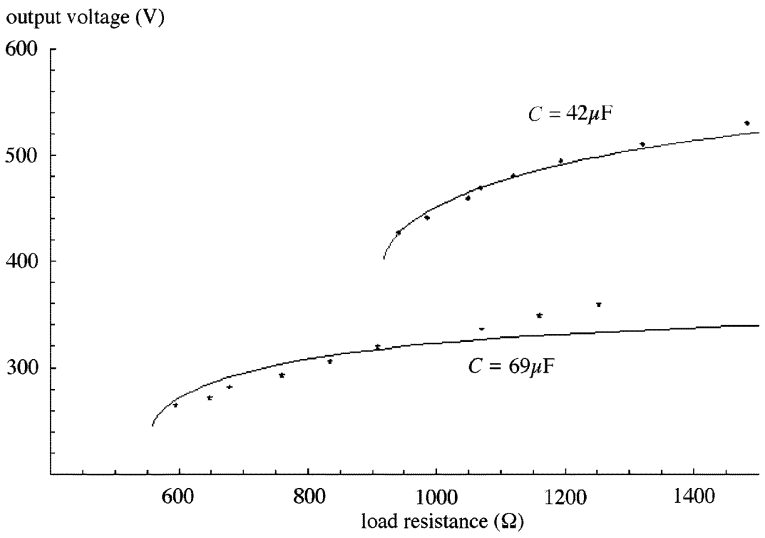

(c)

Fig. 7. Measured stability boundaries taken at $V_{\text {in }}=100 \mathrm{~V}(\mathrm{rms})$ and $50 \mathrm{~Hz}$ line frequency. Experimental data points are plotted as dots. Solid curves are from analytical expressions. (a) $C=69 \mu \mathrm{F}$ and $\tau_{F}=8.46 \mathrm{~ms}$; (b) $C=69 \mu \mathrm{F}$ and $G_{F}=17.46 \mathrm{~A}$; (c) $G_{F}=17.46 \mathrm{~A}$ and $\tau_{F}=8.46 \mathrm{~ms}$. Note that the y axis would have been $v_{o} / V_{\text {in }}$ for better generality, as shown in our analytical study. However, for measurement convenience, we have fixed the input voltage in our experiments and varied the output voltage.

Hertz. For instance, $\tau_{F}=1 / 2 \pi(50) \mathrm{s}=3.18 \mathrm{~ms}$ refers to a time constant equal to the period of a $50 \mathrm{~Hz}$ signal.

4) We have performed the experiments for relatively small output capacitor values in order to allow instability to be seen with the usual gain values readily implementable by the particular controller UC3854A. However, we should stress that the phenomenon remains invariant for practical capacitor values (e.g., hundreds of $\mu \mathrm{F}$ ) provided that higher feedback gain values are used accordingly, as per Fig. 4 or (36).

\section{CONCLUSION}

At present, the main technique for the analysis of power converters is based on averaging and subsequent small-signal linearization. Since PFC converters are subject to a time-varying input voltage and the current-shaping process is nonlinear, the results obtained from small-signal linear analysis have limited validity and often provide inaccurate stability information, as has been explained and demonstrated by Orabi et al. [11]-[13] with computer simulations and laboratory measurements. Nonlinear analytical models would be desirable for stability evaluation. This paper has applied a method of double averaging for modeling PFC converters, leading to nonlinear models from which closed-form stability conditions can be derived. This method is capable of revealing the low-frequency stability of the circuit. For instability near the switching frequency, moreover, we need a different type of models, such as those based on discrete-time mapping [15], [22], for revealing the fast-scale instability.

\section{ACKNOWLEDGMENT}

The authors wish to express their gratitude to the reviewers for drawing their attention to [6] and [14], and to one reviewer for clarifying the issue of omitting the inductor current dynamics.

\section{REFERENCES}

[1] L. H. Dixon, Jr., "High power factor preregulator for off-line power supplies," in Unitrode Switching Regulated Power Supply Design Manual. Merrimack, NH: Unitrode Corp., 1990.

[2] R. Redl, "Power-factor-correction in single-phase switching-mode power supplies-An overview," Int. J. Electron., vol. 77, no. 5, pp. 555-582, 1994.

[3] P. C. Stefanov and A. M. Stanković, "Modeling of UPFC operation under unbalanced conditions with dynamic phasors," IEEE Trans. Power Syst., vol. 17, no. 2, pp. 395-403, May 2002.

[4] A. M. Stanković, S. R. Sanders, and T. Aydin, "Dynamic phasors in analysis of unbalanced polyphase ac machines," IEEE Trans. Energy Convers., vol. 17, no. 1, pp. 107-113, Mar. 2002. 
[5] D. Maksimović, A. M. Stanković, V. J. Thottuvelil, and G. C. Verghese, "Modeling and simulation of power electronic converters," Proc. IEEE, vol. 89, no. 6, pp. 898-912, Jun. 2001.

[6] V. J. Thottuvelil, D. Chin, and G. Verghese, "Hierachical approaches to modeling high-power-factor AC-DC converters," IEEE Trans. Power Electron., vol. 6, no. 2, pp. 179-187, Apr. 1991.

[7] G. Zhu, H. Wei, P. Kornetzky, and I. Batarseh, "Small-signal modeling of a single-switch AC/DC power factor correction circuit," in IEEE Power Electron. Specialists Conf. Rec., Fukuoka, Japan, 1998, pp. 601-607.

[8] G. Zhu, C. Iannello, P. Kornetzky, and I. Batarseh, "Large-signal modeling of a single-switch power factor correction converter," in IEEE Power Electron. Specialists Conf. Rec., Galway, Ireland, 2000, pp. 1351-1357.

[9] V. S. Murali, C. K. Tse, and M. H. L. Chow, "Small-signal analysis of single-stage cascaded boost-and-buck PFC converters," in IEEE Power Electron. Specialists Conf. Rec., Fukuoka, Japan, 1998, pp. 608-614.

[10] M. Shen and Z. Qian, "A unified average model for single stage and two stage power factor correction converters," in Proc. Appl. Power Electron. Conf. Expo., 2001, pp. 125-129.

[11] M. Orabi, T. Ninomiya, and C. Jin, "Nonlinear dynamics and stability analyzes of boost power-factor-correction circuit," in Proc. Int. Conf. Power Syst. Tech., 2002, pp. 600-605.

[12] _ _A novel modeling of instability phenomena in PFC converter," in Proc. IEEE Int. Telecommun. Energy Conf., 2002, pp. 566-573.

[13] M. Orabi and T. Ninomiya, "Nonlinear dynamics of power-factor-correction converter," IEEE Trans. Ind. Electron., vol. 50, no. 6, pp. $1116-1125$, Dec. 2003

[14] F. Gordillo, G. Escobar, and J. Aracil, "Bifurcation analysis of a power factor precompensator," in Stability and Stabilization of Nonlinear Systems. London, U.K.: Springer-Verlag, 1999.

[15] H. H. C. Iu, Y. Zhou, and C. K. Tse, "Fast-scale instability in a PFC boost converter under average current mode control," Int. J. Circuit Theory Appl., vol. 31, no. 6, pp. 611-624, 2003

[16] X. Wu, C. K. Tse, O. Dranga, and J. Lu, "Fast-scale instability of single-stage power-factor-correction power supplies," IEEE Trans. Circuits Syst. I, vol. 53, no. 1, pp. 204-213, Jan. 2006.

[17] R. D. Middlebrook and S. Ćuk, "A general unified approach to modeling switching-converter power stages," in IEEE Power Electron. Spec. Conf. Rec., Pasadena, CA, 1976, pp. 18-34.

[18] C. K. Tse, Y. S. Lee, and W. C. So, "An approach to modeling DC-DC converters using graph theoretic concepts," Int. J. Circuit Theory Appl., vol. 21 , no. 4, pp. 371-384, 1993.

[19] S. R. Sanders, J. M. Noworolski, X. Z. Liu, and G. Verghese, "Generalized averaging method for power electronic circuits," IEEE Trans. Power Electron., vol. 6, no. 2, pp. 251-259, Mar. 1991

[20] S. Banerjee and G. Verghese, Eds., Nonlinear Phenomena in Power Electronics: Attractors, Bifurcations, Chaos and Nonlinear Control. New York: IEEE Press, 2001.

[21] C. K. Tse, Complex Behavior of Switching Power Converters. Boca Raton, FL: CRC Press, 2003.

[22] O. Dranga, C. K. Tse, H. H. C. Iu, and I. Nagy, "Bifurcation behavior in power-factor-correction boost converter," Int. J. Bifurc. Chaos, vol. 13, no. 10 , pp. 3107-3114, 2003.

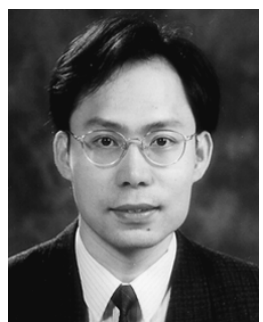

Siu-Chung Wong (M'01) received the B.Sc. degree in physics from the University of Hong Kong, Hong Kong, the M.Phil. degree in electronics from the Chinese University of Hong Kong, Hong Kong, and the $\mathrm{Ph} . \mathrm{D}$. degree from the University of Southampton, Southampton, U.K., in in 1986, 1989, and 1997 , respectively.

He is currently an Assistant Professor in the Department of Electronic and Information Engineering at Hong Kong Polytechnic University, Hong Kong. His research interests include modeling of power converters, nonlinear analysis of power electronics and Internet traffic analysis.

Dr. Wong is a member of the Electrical College, The Institution of Engineers, Australia.

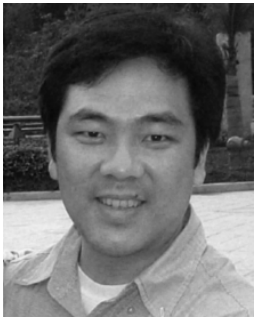

Chi K. Tse (M'90-SM'97-F'06) received the B.Eng. (Hons) degree with first class honors in electrical engineering and the $\mathrm{Ph} . \mathrm{D}$. degree from the University of Melbourne, Melbourne, Australia, in 1987 and 1991, respectively.

He is presently Chair Professor of Electronic Engineering at the Hong Kong Polytechnic University, Hong Kong, and his research interests include chaotic dynamics and power electronics. He is the author of Linear Circuit Analysis (Reading, MA: AddisonWesley, 1998) and Complex Behavior of Switching Power Converters (Boca Raton, FL: CRC Press, 2003), and co-author of ChaosBased Digital Communication Systems (New York: Springer-Verlag, 2003) and Signal Reconstruction With Applications to Chaos-Based Communications (Beijing, China: Tsinghua University Press, 2005), and co-holder of a U.S. patent.

Dr. Tse served as an Associate Editor for the IEEE TRANSACTIONS ON CirCUITS AND SYSTEMS-I: FundAMENTAL THEORY AND APPLICATIONS from 1999 to 2001, and since 1999, he has been an Associate Editor for the IEEE TRANSACTIONS ON POWER ELECTRONICS. He currently also serves as an Associate Editor for the International Journal of Systems Science, a Guest Associate Editor of the IEICE Transactions on Fundamentals of Electronics, Communications and Computers, and a Guest Editor of Circuits, Systems and Signal Processing. He was awarded the L.R. East Prize by the Institution of Engineers, Australia, in 1987. He won the IEEE TRANSACTIONS ON POWER ELECTRONICS Prize Paper Award for 2001 and the International Journal of Circuit Theory and Applications Best Paper Award for 2003. In 2005, he was appointed an IEEE Distinguished Lecturer. While with Hong Kong Polytechnic University, he twice received the President's Award for Achievement in Research, the Faculty's Best Researcher Award, the Research Grant Achievement Award, and a few other teaching awards.

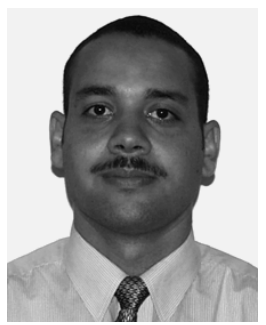

Mohamed Orabi (S'02-M'04) was born in Kena, Egypt, in 1974. He received the B.Sc. and M.Sc. degrees in electrical engineering from Elminia University, Elminia, Egypt, in 1996 and 2000, respectively, and the $\mathrm{Ph} . \mathrm{D}$. degree in power electronics from Kyushu University, Fukuoka, Japan, in 2004.

He has been with the Department of Electrical Engineering, Aswan Faculty of Engineering, South Valley University, Aswan, Egypt, first as an Administrator from 1996 to 2000, then as Research Assistant from 2000 to 2004, and as an Assistant Professor since 2004. His research interests include developing and designing switched-mode power converters, applications to power factor correction, stability problems, nonlinear phenomena, chaos, and period doubling bifurcation. He has authored more than 30 published papers.

Dr. Orabi received the 2002 Excellent Student Award of the IEEE Fukuoka Section, the Best Paper Award of 28th Annual Conference of the IEEE Industrial Electronics Society in 2002, the IEEE IES Student Grant from 2003 IEEE International Symposium on Industrial Electronics, and the IEICE Best Young Researcher Award for 2004.

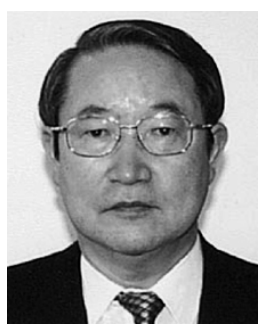

Tamotsu Ninomiya (M'89-SM'98-F'01) received the B.E., M.E., and Dr. Eng. degrees in electronics from Kyushu University, Fukuoka, Japan, in 1967, 1969 , and 1981, respectively.

Since 1969, he has been with the Department of Electronics, Kyushu University, first as a Research Assistant and, since 1988, as a Full Professor. Since the reorganization in 1996, he has been a Professor in the Department of Electrical and Electronic Systems Engineering of the Graduate School of Information Science and Electrical Engineering. He is a specialist in the field of power electronics, including the analysis of switching power converters and their electromagnetic interference problems, the development of noise-suppression techniques, and piezoelectric transformer converters. He has authored about 200 published technical papers.

Prof. Ninomiya has served as a Member of the Program Committee and Session Chairman of the IEEE Power Electronics Specialist Conference (PESC) sponsored by the IEEE Power Electronics Society (PELS) for many years. He was Program Vice-Chairman for the 1988 PESC and General Chairman for the 1998 PESC. He was a Member of the Administrative Committee of the IEEE PELS from 1993 through 1998. He served as Chairman of the Professional Group on High-Frequency Applied Magnetics of the Institute of Electrical Engineers of Japan, and as Chairman of the Technical Group on Power Engineering in Electronics and Communications of the Institute of Electronics, Information, and Communication Engineers of Japan. 